\title{
ANDES

www.scielo.cl

\section{Efecto de la extensión de licencia maternal en la lactancia materna según pobreza en Chile 2008-2018}

\author{
Maternal leave extension effect on breastfeeding according to poverty, \\ Chile 2008-2018
}

\author{
Patricia Caro $^{\oplus a}$, Carolina Vidal $^{\oplus a}$, Estrella Rojas ${ }^{\oplus b}$, Camila Zárate ${ }^{\oplus b}$, Carlos Sandaña ${ }^{\oplus a}$
}

aEscuela de Salud Pública, Facultad de Ciencias, Universidad Mayor. Santiago, Chile

'Escuela de Nutrición y Dietética, Universidad Tecnológica de Chile INACAP. Santiago, Chile

Recibido: 01 de octubre de 2020; Aceptado: 16 de enero de 2021

\section{¿Qué se sabe del tema que trata este estudio?}

La implementación de la ley que extiende el postnatal de 12 a 24 semanas en Chile en el año 2011 ha provocado un aumento en la prevalencia de lactancia materna exclusiva al sexto mes de vida.

\begin{abstract}
¿Qué aporta este estudio a lo ya conocido?
El aumento en la prevalencia de lactancia materna exclusiva al sexto mes es mayor en el grupo de menor pobreza, sin embargo, el grupo de mayor pobreza mantiene prevalencias más altas comparado a los otros grupos al final del periodo.
\end{abstract}

\section{Resumen}

El año 2011 se extendió la licencia maternal postnatal en Chile de 12 a 24 semanas. Objetivo: Determinar el efecto de la extensión de la licencia maternal en la prevalencia de lactancia materna en Chile, según grupos de pobreza. Método: Estudio ecológico sobre la prevalencia anual de lactancia materna exclusiva al $1^{\circ}$ mes y $6^{\circ}$ mes y complementada al $12^{\circ}$ mes en el sistema público de salud entre $2008 \mathrm{y}$ 2018, en base a los registros estadísticos mensuales de la atención primaria. Se calculó la prevalencia a nivel país y por grupos de regiones según el nivel de pobreza reportado por la Encuesta de Caracterización Socioeconómica Nacional (CASEN). Se determinó la variación en la tendencia temporal mediante modelos de regresión de Poisson segmentados (joinpoint), estimando los porcentajes de cambio anual. Resultados: A nivel nacional, las prevalencias de lactancia materna exclusiva al $6^{\circ}$ mes y complementada a los 12 meses aumentaron 5,9\% y 4,2\% anualmente desde 2011, alcanzando un $58,9 \%$ y $40,2 \%$ en 2018 , respectivamente. Este patrón se repite en los diferentes grupos de pobreza regional, con mayores prevalencias en el grupo más pobre, alcanzando $61,7 \%$ al $6^{\circ}$ mes y $51,6 \%$ a los 12 meses en 2018. La lactancia materna exclusiva al mes disminuyó 1,7\% anual entre 2008 y 2014, para luego mantenerse, alcanzando un $74,4 \%$ en 2018. Conclusiones: La prevalencia de lactancia materna exclusiva al $6^{\circ}$ mes aumentó significativamente a partir del año 2011, con la extensión de la licencia maternal. Este efecto se produce en todos los grupos regionales de pobreza, estrechando las diferencias en la prevalencia.
Palabras clave: Lactancia Materna;

Prevalencia;

Licencia Maternal;

Pobreza;

Chile 


\begin{abstract}
In Chile in 2011, the postnatal leave was extended from 12 to 24 weeks. Objective: To determine the effects of the extension of maternity leave on the prevalence of breastfeeding in Chile according to poverty groups. Methods: Ecological study on the annual prevalence of exclusive breastfeeding at 1st month (EBF1m) and 6th month (EBF6m), and supplemented at 12th month (BF12m) in the public health system between 2008 and 2018, based on the monthly statistical records of primary care. The prevalence was calculated nationally and by region groups according to the poverty level reported by the National Socioeconomic Characterization Survey (CASEN). The variation in the time trend was determined through segmented Poisson regression models (joinpoint), estimating the Annual Percentage Change (APC). Results: Nationally, since 2011, the prevalence of EBF6m and BF $12 \mathrm{~m}$ increased annually by $5.9 \%$ and $4.2 \%$, reaching $58.9 \%$ and $40.2 \%$ in 2018 , respectively. This pattern is repeated in the different regional poverty groups, showing a higher prevalence in the poorest group, reaching $61.7 \%$ at EBF6m and $51.6 \%$ at BF $12 \mathrm{~m}$ in 2018 . The EBF1m decreased annually by $1.7 \%$ between 2008 and 2014 and then remained without changes, reaching $74.7 \%$ in 2018. Conclusions: The EBF6m prevalence increased significantly since 2011 due to the extension of the maternity leave. This effect occurs in all regional groups of poverty, narrowing the differences in prevalence.
\end{abstract}

Keywords:

Breastfeeding;

Prevalence;

Maternity Leave;

Poverty;

Chile

\section{Introducción}

A partir del año 1990, Chile implementa como parte de sus políticas públicas la creación del Hospital amigo de la madre y el niño, el Programa "Chile Crece Contigo" (Ley 20.379) y la modificación del decreto $\mathrm{N}^{\circ} 977$ del Código Sanitario prohibiendo la publicidad de alimentos sucedáneos de la leche materna en el año 2017, entre otras actividades orientadas al fomento de la lactancia materna ${ }^{1}$. En el año 2011, siguiendo la recomendación 191 de la Organización Internacional del Trabajo $(\mathrm{OIT})^{2}$, Chile promulga la ley 20.545, la cual incorpora una extensión de 12 semanas una vez finalizada la licencia maternal (12 semanas), quedando un periodo de 24 semanas para el reposo en el periodo postnatal.

Un estudio previo que evaluó el efecto de la extensión de la licencia maternal sobre la lactancia materna exclusiva al sexto mes en Chile evidenció un aumento de 3,6\% en el periodo $2011-2013^{3}$. A la fecha, el efecto desagregado por niveles de pobreza regional no se ha estudiado a pesar de que la evidencia ha mostrado que la lactancia materna representa un problema de inequidad en salud, ya que las poblaciones con menor nivel socioeconómico y educacional tienden a tener menos probabilidades de amamantar y/o de iniciar la lactancia en las primeras horas de vida del recién nacido en comparación a sus pares de mejor nivel socioeconómico y educacional ${ }^{4,5}$. Sin embargo, una vez iniciada la lactancia materna son las poblaciones de menor nivel socioeconómico quienes muestran mayor prevalencia de lactancia materna en todas las edades ${ }^{4,6}$.

Esta inequidad se ha asociado al género, ya que son las mujeres quienes sobrellevan una alta carga de pobreza a nivel mundial, a su vez, las mujeres de bajo nivel socioeconómico tienen menos ayudas formales e informales para iniciar la lactancia materna, sumado a mayores cargas de trabajo junto al cuidado infantil y un mayor nivel de desinformación respecto al tema ${ }^{7,8}$.

Se ha observado que los niños que no son amamantados tienen un mayor riesgo de mortalidad, y mayores consultas por enfermedades respiratorias y gastrointestinales, sumado a una mayor prevalencia de maloclusiones dentales y la probabilidad de tener sobrepeso y obesidad en la vida adulta ${ }^{9-15}$. Mientras que, en la madre, el no dar de amamantar a su hijo se ha asociado al cáncer de mama, cáncer de ovario, diabetes mellitus tipo 2 y osteoporosis ${ }^{16,17}$. Esto tiene especial importancia, considerando que estas enfermedades están dentro de las principales causas de morbilidad y mortalidad entre las mujeres chilenas ${ }^{18}$. Por estas razones, es que la lactancia materna constituye una de las intervenciones en salud más costo-efectivas, protegiendo la salud de la madre y el hijo ${ }^{4}$.

La Organización Mundial de la Salud (OMS) recomienda iniciar la lactancia materna exclusiva (LME) dentro de las primeras horas de vida hasta los 6 meses, para luego ser complementada con alimentos sólidos hasta los 2 años o más de vida ${ }^{19,20}$. Sin embargo, a nivel mundial, sólo un 38\% de los niños menores de 6 meses son amamantados de manera exclusiva ${ }^{21}$.

El objetivo de esta investigación es analizar el efecto de la implementación de la ley que extiende la licencia maternal de 12 a 24 semanas sobre la lactancia materna exclusiva al sexto mes y complementada al duodécimo mes, desagregando por niveles de pobreza. 


\section{Métodos}

Estudio observacional ecológico para determinar la tendencia de la prevalencia de lactancia materna al $1^{\circ}$, $6^{\circ}$ y $12^{\circ}$ mes de vida en Chile en el periodo 2008-2018. Se estimó la prevalencia de lactancia materna exclusiva $1^{\circ}$ mes (LME1m) y $6^{\circ}$ mes (LME6m) y complementada al $12^{\circ}$ mes (LM12m) en niños bajo control en el servicio público de salud, entendiéndose lactancia materna exclusiva cuando el niño es amamantado solamente con leche materna, sin recibir ningún otro tipo de sustituto lácteo ni alimento, mientras que lactancia materna complementada es aquella en la que el niño recibe lactancia materna además de otros alimentos sólidos y/o líquidos.

Se calcularon las prevalencias de LME1m, LME6m y LM12m utilizando como numerador la cantidad de niños amamantados y como denominador el número de niños bajo control en el sistema público de salud de Chile, según región del país. Estos datos se obtuvieron a partir del Registro Estadístico Mensual (REM) emitido en la red pública de atención de salud y disponible en el Departamento de Información y Estadística en Salud (DEIS). Este registro contiene la información de lactancia materna autorreportada por la madre a los profesionales de la salud mientras realizan el control del niño sano al $1^{\circ}, 6^{\circ}$ y $12^{\circ}$ mes de vida. Los registros mensuales se consolidaron para obtener cifras anuales de lactancia materna a nivel país y por región para cada año de estudio. Se utilizó la regionalización decretada el año 2007, la cual divide a Chile en 15 regiones (Leyes 20.174 y 20.175).

Con el objetivo de describir eventuales diferencias según nivel de pobreza en el país, la totalidad de las regiones de Chile se agruparon en 4 grupos de pobreza utilizando la siguiente metodología: Para cada región del país se calculó el promedio de la incidencia de personas en situación de pobreza según nivel de ingresos, reportados por las Encuestas de Caracterización Socioeconómica (CASEN) de los años 2009, 2011, 2013, 2015 y $2017^{22}$. Luego se estimaron los percentiles 25, 50 y 75 del promedio de la incidencia de pobreza por región, con el fin de crear 4 grupos, los cuales quedaron conformados de la siguiente manera: grupo 1 (menos pobre), regiones de Magallanes y la Antártica Chilena, de Antofagasta, de Aysén del General Carlos Ibañez del Campo y Metropolitana de Santiago; grupo 2, regiones de Atacama, de Tarapacá, de Arica y Parinacota y de Valparaíso; grupo 3, regiones del Libertador General Bernardo O'Higgins, de Coquimbo, de Los Lagos y del Biobío; y grupo 4 (más pobre), regiones de Los Ríos, del Maule y de La Araucanía.

Se utilizaron modelos de regresión de Poisson segmentada (regresión joinpoint) para estimar el porcentaje de cambio anual (PCA) en la prevalencia de lactancia materna para el periodo de estudio, junto con sus intervalos de confianza de un $95 \%$, considerando estadísticamente significativo un $\mathrm{p}<0,05$. Los análisis se realizaron para cada mes de lactancia materna evaluado, tanto a nivel país como por grupo de pobreza regional. Los análisis fueron realizados mediante el software Jointpoint Regression Program 4.8.0.1.

\section{Resultados}

La prevalencia de LME1m en la población atendida en el sistema público de Chile fue de $82,9 \%$ en 2008, disminuyendo progresivamente hasta un $73,6 \%$ en el año 2017, con una leve alza en 2018. Este patrón es similar en los diferentes grupos de pobreza regionales, destacando que el grupo con mayor pobreza presenta prevalencias de LME1m superiores a los otros grupos en todos los años de estudio (tabla 1).

Por su parte, se obtuvo una prevalencia de LME6m de $49,1 \%$ en 2008 a nivel país, la que disminuyó $41,1 \%$ en el año 2011. A partir de este año se observa un aumento progresivo hasta alcanzar un $58,9 \%$ en el año 2018. Durante todo el periodo, las mayores cifras de prevalencia las presentó el grupo de mayor pobreza y las menores cifras el grupo de menor pobreza regional. La diferencia entre estos dos grupos alcanzaba 11,2 puntos porcentuales en 2008, disminuyendo a 3,8 puntos porcentuales en 2018 (tabla 1).

La prevalencia de LM12m a nivel nacional presentó una disminución desde $39,3 \%$ en 2008 a un mínimo de $29,8 \%$ en 2011. A partir de esta fecha, las cifras aumentan alcanzando un $40 \%$ en 2017 y 2018. Esta disminución y posterior aumento a lo largo del periodo se evidencia en todos los grupos de pobreza regional, aunque aquellos de mayor pobreza presentan mayores prevalencias de LM12m que los grupos con menor pobreza regional, alcanzando cifras de $42,1 \%$ en el grupo 3 y de 51,6\% en el grupo 4 durante el año 2018 (tabla 1).

Las prácticas de LME6m y LM12m muestran un aumento significativo desde el año 2011 en adelante. En el caso de la prevalencia de LME6m, presentó una tendencia al aumento en 5,9\% anual (IC95\%: 3,9-8,0) desde el año 2011, mientras que LM12m este aumento fue de 4,2\% anual (IC95\%: 1,4-7,0). En ambos casos, entre los años 2008 y 2011 la tendencia no presentó cambios significativos (PCA: -5,9; IC95\%: -13,2-2,1 para LME6m y PCA: -8,7; IC95\%: $-17,4-1,0$ para LM12m). Por el contrario, la LME1m presentó una disminución de 1,7\% anual entre 2008 y 2014 (PCA: -1,7; IC95\%: -2,3--1,2), sin presentar variaciones significativas entre 2014 y 2018 (PCA: 0,1; IC95\%: $-1,3-1,5)$ (figura 1). 
Tabla 1. Prevalencia anual de LME1m, LME6m y LM12m a nivel país y según grupo de pobreza regional. Chile, 2008-2018

\begin{tabular}{llllllllllll}
\hline & 2008 & 2009 & 2010 & 2011 & 2012 & 2013 & 2014 & 2015 & 2016 & 2017 & 2018 \\
\hline & & & & & \multicolumn{2}{c}{ Prevalencia de LME1m (\%) } & & & \\
Grupo 1 & 81,3 & 79,3 & 77,7 & 77,0 & 76,5 & 75,8 & 72,8 & 72,6 & 72,8 & 72,9 & 73,6 \\
Grupo 2 & 80,0 & 79,2 & 77,8 & 75,1 & 73,6 & 73,8 & 71,8 & 72,9 & 74,3 & 73,1 & 74,7 \\
Grupo 3 & 85,6 & 80,4 & 79,0 & 77,4 & 75,6 & 75,5 & 73,7 & 74,0 & 73,1 & 73,9 & 74,9 \\
Grupo 4 & 86,3 & 83,0 & 78,6 & 81,1 & 80,0 & 79,6 & 79,1 & 78,0 & 76,1 & 75,6 & 75,7 \\
Total país & 82,9 & 80,0 & 78,2 & 77,3 & 76,2 & 75,9 & 73,7 & 73,8 & 73,6 & 73,6 & 74,4 \\
& & & & & Prevalencia de LME6m (\%) & & & \\
Grupo 1 & 45,1 & 38,8 & 33,9 & 33,4 & 42,8 & 43,6 & 43,0 & 50,8 & 55,0 & 55,9 & 57,9 \\
Grupo 2 & 50,3 & 45,8 & 43,0 & 41,9 & 40,5 & 42,0 & 42,0 & 52,2 & 55,2 & 56,1 & 57,8 \\
Grupo 3 & 48,8 & 45,4 & 44,5 & 41,3 & 43,6 & 46,1 & 46,4 & 54,5 & 58,2 & 57,7 & 60,2 \\
Grupo 4 & 56,3 & 50,9 & 52,1 & 50,8 & 46,5 & 48,4 & 48,5 & 56,8 & 59,5 & 61,8 & 61,7 \\
Total país & 49,1 & 45,1 & 43,5 & 41,1 & 43,1 & 44,7 & 44,6 & 53,0 & 56,6 & 57,3 & 58,9 \\
& & & & & Prevalencia de LM12m (\%) & & & \\
Grupo 1 & 32,9 & 29,4 & 26,7 & 28,6 & 28,6 & 32,9 & 30,2 & 28,2 & 31,4 & 36,4 & 36,9 \\
Grupo 2 & 36,0 & 33,7 & 26,8 & 25,6 & 29,3 & 31,9 & 30,9 & 31,7 & 33,7 & 37,9 & 39,7 \\
Grupo 3 & 43,0 & 41,4 & 38,9 & 33,6 & 34,5 & 37,3 & 37,1 & 35,5 & 38,2 & 43,4 & 42,1 \\
Grupo 4 & 51,5 & 43,6 & 38,2 & 33,7 & 34,9 & 38,3 & 36,1 & 34,8 & 37,7 & 49,4 & 51,6 \\
Total país & 39,3 & 35,7 & 31,7 & 29,8 & 31,4 & 34,8 & 33,2 & 31,9 & 34,7 & 40,3 & 40,2 \\
\hline
\end{tabular}

Grupo 1: menor pobreza regional, Grupo 4: mayor pobreza regional. LME: lactancia materna exclusiva.

Según grupo de pobreza regional, la prevalencia de LME6m presentó una tendencia significativa al aumento desde el año 2010 (grupo 1), 2011 (grupo 3) y 2012 (grupos 2 y 4). Si bien hay diferencias en los valores puntuales de PCA, en base a la magnitud de los intervalos de confianza no es posible afirmar que existan diferencias significativas entre los diferentes grupos (tabla 2).

La prevalencia de LM12m presentó un aumento en los grupos 2 y 4 desde el año 2011 y en el grupo 3 desde el año 2012, sin diferencias en la magnitud de cambio entre estos tres grupos. El grupo de menor pobreza (grupo 1) no presentó variaciones significativas en la prevalencia de LM12m durante el periodo (PCA: 2,0; IC95\%: 0,0-4,0) (tabla 2).

Por el contrario, la prevalencia de LME1m en el grupo de mayor pobreza (grupo 4) presentó una disminución significativa de 1,2\% anual (IC95\%: -1,6--

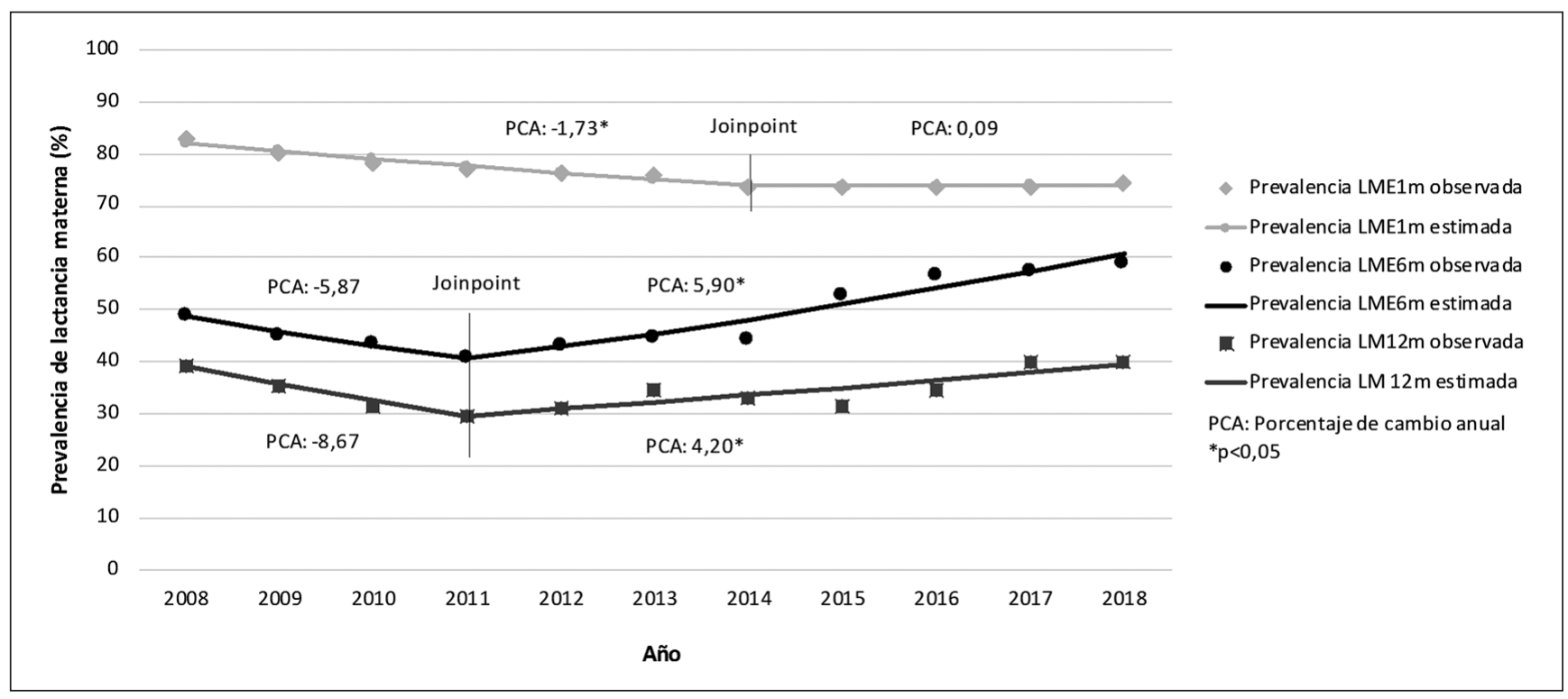

Figura 1. Tendencia de prevalencias de LME1m, LME6m y LM12m. Chile, 2008-2018 (LME: lactancia materna exclusiva). 
Tabla 2. Porcentaje de cambio anual (PCA) en prevalencias de LME1m, LME6m y LM12m, según grupos de pobreza regional. Chile, 2008-2018

\begin{tabular}{|c|c|c|c|}
\hline & Periodo & PCA & (IC95\%) \\
\hline & \multicolumn{3}{|c|}{ Prevalencia de LME1m } \\
\hline Grupo 1 & $\begin{array}{l}2008-2015 \\
2015-2018\end{array}$ & $\begin{array}{c}-1,5 \\
0,4\end{array}$ & $\begin{array}{l}(-1,9 ;-1,1) \\
(-1,6 ; 2,5)\end{array}$ \\
\hline Grupo 2 & $\begin{array}{l}2008-2014 \\
2014-2018\end{array}$ & $\begin{array}{c}-1,8 \\
1,1\end{array}$ & $\begin{array}{l}(-2,4 ;-1,2) \\
(-0,4 ; 2,5)\end{array}$ \\
\hline Grupo 3 & $\begin{array}{l}2008-2012 \\
2012-2018\end{array}$ & $\begin{array}{l}-2,9 \\
-0,3\end{array}$ & $\begin{array}{l}(-4,2 ;-1,6) \\
(-1,4 ; 0,7)\end{array}$ \\
\hline \multirow[t]{2}{*}{ Grupo 4} & 2008-2018 & $-1,2$ & $(-1,6 ;-0,7)$ \\
\hline & \multicolumn{3}{|c|}{ Prevalencia de LME6m } \\
\hline Grupo 1 & $\begin{array}{l}2008-2010 \\
2010-2018\end{array}$ & $\begin{array}{c}-10,3 \\
6,6\end{array}$ & $\begin{array}{c}(-37,3 ; 28,1) \\
(4,4 ; 8,9)\end{array}$ \\
\hline Grupo 2 & $\begin{array}{l}2008-2012 \\
2012-2018\end{array}$ & $\begin{array}{c}-5,0 \\
7,1\end{array}$ & $\begin{array}{c}(-11,0 ; 1,4) \\
(3,9 ; 10,5)\end{array}$ \\
\hline Grupo 3 & $\begin{array}{l}2008-2011 \\
2011-2018\end{array}$ & $\begin{array}{l}-5,1 \\
5,9\end{array}$ & $\begin{array}{c}(-10,2 ; 0,3) \\
(3,8 ; 8,0)\end{array}$ \\
\hline \multirow[t]{2}{*}{ Grupo 4} & $\begin{array}{l}2008-2012 \\
2012-2018\end{array}$ & $\begin{array}{c}-4,3 \\
5,5\end{array}$ & $\begin{array}{c}(-10,1 ; 1,9) \\
(2,9 ; 8,2)\end{array}$ \\
\hline & \multicolumn{3}{|c|}{ Prevalencia de LM12m } \\
\hline Grupo 1 & $2008-2018$ & 2,0 & $(0,0 ; 4,0)$ \\
\hline Grupo 2 & $\begin{array}{l}2008-2011 \\
2011-2018\end{array}$ & $\begin{array}{c}-10,0 \\
5,9\end{array}$ & $\begin{array}{c}(-17,2 ;-2,2) \\
(3,6 ; 8,3)\end{array}$ \\
\hline Grupo 3 & $\begin{array}{l}2008-2012 \\
2012-2018\end{array}$ & $\begin{array}{l}-5,7 \\
3,6\end{array}$ & $\begin{array}{c}(11,0 ;-0,2) \\
(0,7 ; 6,7)\end{array}$ \\
\hline Grupo 4 & $\begin{array}{l}2008-2011 \\
2011-2018\end{array}$ & $\begin{array}{c}-14,5 \\
5,8\end{array}$ & $\begin{array}{c}(-26,7 ;-0,2) \\
(1,1 ; 10,8)\end{array}$ \\
\hline
\end{tabular}

Grupo 1: menor pobreza regional, Grupo 4: mayor pobreza regional.

$0,7)$ durante el periodo estudiado. En los otros grupos de pobreza también se observó una disminución en la prevalencia de LME1m, pero no durante todo el periodo; el grupo 1 disminuyó hasta el año 2015, el grupo 2 hasta 2014 y el grupo 3 hasta el año 2012. No se detectaron diferencias significativas en la magnitud del PCA entre los diferentes grupos (tabla 2).

\section{Discusión}

La prevalencia de lactancia materna exclusiva a los 6 meses ha aumentado a nivel país a partir del año 2011 en todos los grupos de pobreza regional, considerando la temporalidad es altamente probable que estos hallazgos se relacionen con la promulgación de la ley de permiso postnatal parental. La implementación de esta ley permite que las madres puedan permanecer con sus hijos una mayor cantidad de tiempo gozando de una licencia remunerada, lo cual va en concordancia con los acuerdos internacionales que protegen la salud de la madre y el niño, pues el reintegro laboral es uno de los principales factores causantes del cese de la lactancia materna ${ }^{23-26}$.

Se estima que en 2019 el 44\% de los menores de 6 meses recibieron lactancia materna exclusiva a nivel mundial y que en Latinoamérica esta cifra alcanzó un $33 \%$, destacando Perú con la prevalencia más alta $(69,8 \%)^{27}$. Considerando este panorama, Chile en el año 2018 se encuentra casi 15 puntos porcentuales por sobre la estimación mundial.

Al final del periodo se observa una brecha en la prevalencia de LME6m de casi 4 puntos porcentuales entre los grupos de mayor y menor pobreza, brecha que alcanza casi 15 puntos porcentuales en la prevalencia de LM12m. Esto podría relacionarse con el efecto que tiene la extensión del periodo postnatal en la lactancia materna en los niveles socioeconómicos altos, considerando que la ley beneficia a las madres trabajadoras formales. En Chile la ocupación laboral femenina es directamente proporcional a los años de escolaridad, observando que en las mujeres con nivel primaria sólo un $31,2 \%$ está ocupada en comparación al $87 \%$ de ocupación en mujeres con postgrado y en relación a la formalidad laboral, el 35,5\% de las mujeres son trabajadoras informales, es decir son trabajadoras sin contrato o independientes sin cotizaciones previsionales vigentes ${ }^{28,29}$.

Si bien la OMS recomienda la extensión de la licencia maternal como un mecanismo para promover la lactancia materna, la evidencia no ha mostrado una relación directa entre la duración de la licencia maternal y la lactancia materna ${ }^{20}$. En el estudio de Teurich et al., se observa que Suecia (39\%), Bélgica (28\%) y España $(18 \%)$ tienen las prevalencias más altas de lactancia materna exclusiva al $6^{\circ}$ mes en relación con otros países europeos ${ }^{30}$. Al revisar el tiempo de licencia maternal según lo registra la Organización Internacional del Trabajo (OIT) se observa que Suecia otorga 32 semanas, Bélgica 15 semanas y España 16 semanas $^{31}$.

$\mathrm{Al}$ igual que para LME6m, la prevalencia de LM12m también aumentó a nivel país desde el año 2011, aunque en menor medida ( $4,2 \%$ anual) y alcanzando una cifra de $40,2 \%$ en 2018 . La relación entre el aumento en LM12m y la Ley de permiso postnatal parental es menos evidente, considerando que se ha observado una correlación moderada entre la lactancia materna exclusiva y la lactancia mantenida hasta el año de vida en países de bajos y medianos ingresos 4 .

La evidencia ha mostrado que la continuidad de las prácticas de amamantamiento luego de la reincorporación laboral de la madre depende de que ella pueda contar con tiempo para amamantar, tener cerca del trabajo lugares de cuidado infantil y contar con un lugar donde sea posible realizar la extracción de leche materna, entre otros ${ }^{32}$. Según la Encuesta Nacional de 
Lactancia Materna en la Atención Primaria de Chile (ENALMA 2013), un 10,4\% de las madres entrevistadas abandonó la lactancia al reintegrarse al trabajo formal y $41,9 \%$ por problemas asociados a la mala técnica de amamantamiento ${ }^{26}$.

No se detectó un aumento significativo en la prevalencia de LM12m en el grupo regional con menor pobreza, a diferencia de los otros tres grupos. Esto podría vincularse a una relación directa entre el nivel de ingresos y la probabilidad de que las madres comiencen a darle sustitutos de leche materna a sus hijos. En los países de altos ingresos se ha observado que menos de 1 de cada 5 niños son amamantados hasta los 12 meses $^{4}$.

Al inicio del periodo, la prevalencia de LME1m se encontraba sobre el $80 \%$ a nivel país y en todos los grupos regionales de pobreza, mientras que al final del periodo de estudio esta era de $74,4 \%$ en el grupo más pobre y 73,6\% en el grupo menos pobre. A pesar de esta disminución, las cifras de prevalencia observadas en Chile son similares a las reportadas por los países europeos, los cuales rondan el $70 \%{ }^{33}$.

La prevalencia de LME1m disminuyó significativamente a nivel país hasta 2014, pero se ha mantenido sin cambios significativos entre 2014 y 2018, sin observarse que la implementación de la ley haya influido positivamente en la lactancia durante el primer mes de vida. Aunque con variaciones en el año de estabilización, este patrón se repite en los grupos de pobreza regional, excepto en el grupo con mayor pobreza, en el que la prevalencia de LME1m disminuye a lo largo de todo el periodo. Este resultado concuerda con lo referido en la literatura, en donde las mujeres más pobres son más susceptibles a las barreras que dificultan el inicio temprano de la lactancia materna ${ }^{6,34}$, sin embargo, una vez iniciada esta, tienden a amamantar por más tiempo que su contraparte de mayores ingresos ${ }^{33}$, a pesar de la falta de apoyos formales e informales para la lactancia exitosa, a la sobrecarga de trabajo relacionada al cuidado infantil y el poco acceso a información sobre lactancia materna que tienen ${ }^{35}$.

Las limitaciones de este estudio son la dificultad de conseguir información de lactancia materna del sistema privado de salud en Chile (20\% de la población), ya que no existe un registro adecuado y público de esta información. Además, al ser un estudio poblacional, el nivel de pobreza se midió a nivel regional sin conside- rar los efectos que pudiesen existir a nivel individual. En este mismo sentido, la pobreza se distribuye de manera desigual en cada región, efecto que no podemos medir en esta investigación.

En conclusión, la ley de extensión de la licencia postnatal en Chile se relaciona con un aumento en la prevalencia de LME6m, sin embargo, este efecto es menos significativo en los grupos más pobres. Una de las razones que podría explicar este hallazgo es que en los grupos más pobres el trabajo se realiza con mayor frecuencia de manera informal, por lo cual, la implementación de esta ley no sería un beneficio para aquellas madres. Por esta razón, es necesario contar con más estudios que profundicen en los determinantes estructurales e intermedios que median la relación entre las prácticas de amamantamiento y la pobreza en Chile, ya que la evidencia internacional previa ha demostrado que el inicio tardío de la lactancia materna en mujeres pobres se asocia al determinante estructural del géne$\mathrm{ro}^{36}$.

\section{Responsabilidades Éticas}

Protección de personas y animales: Los autores declaran que los procedimientos seguidos se conformaron a las normas éticas del comité de experimentación humana responsable y de acuerdo con la Asociación Médica Mundial y la Declaración de Helsinki.

Confidencialidad de los datos: Los autores declaran que han seguido los protocolos de su centro de trabajo sobre la publicación de datos de pacientes.

\section{Derecho a la Privacidad y Consentimiento Informa- do: Los autores declaran que la información ha sido obtenida de datos previos en forma anonimizada, por lo cual el Comité de Ética de Investigación en uso de sus facultades, ha eximido de la obtención de un con- sentimiento informado, lo cual consta en el acta res- pectiva.}

\section{Conflicto de intereses}

Los autores declaran no tener conflicto de intereses.

\section{Referencias}

1. Atalah E, Castillo C, Reyes C. Efectividad de un programa nacional de fomento de la lactancia materna en Chile 1993-2002. Arch Latinoam Nutr. 2004;54(4):374-8.
2. World Health Organization (WHO), United Nations Children's Fund (UNICEF). Global breastfeeding scorecard, 2019: increasing commitment to breastfeeding through funding and improved policies and programmes. [Internet]. World Health Organization;
2019. Disponible en: https://apps.who.int/ iris/handle/10665/326049 [consultado y citado el 15 de abril de 2020].

3. Caro Moya P, Guerra Muñoz X. Tendencia de la lactancia materna exclusiva en Chile antes y después de la implementación de la Ley 
postnatal parental. Rev Chil Pediatr. 2018;89(2):190-5.

4. Victora CG, Bahl R, Barros AJ, et al; Lancet Breastfeeding Series Group. Breastfeeding in the 21st century: epidemiology, mechanisms, and lifelong effect. Lancet. 2016 Jan 30;387(10017):475-90.

5. Pérez-Escamilla R, Sellen D. Equity in breastfeeding: Where do we go from here? J Hum Lact. 2015;31(1):12-4.

6. Temple Newhook J, Newhook LA, Midodzi WK, et al. Poverty and Breastfeeding: Comparing Determinants of Early Breastfeeding Cessation Incidence in Socioeconomically Marginalized and Privileged Populations in the FiNaL Study. Health Equity. 2017;1(1):96-102.

7. Duffy A, Mandell N. Poverty in Canada. In: Gender and Women's Studies in Canada. Edited by Hobbs M, Rice C. Toronto: Women's Press. 2013;606-16.

8. Newhook JT, Ludlow V, Newhook LA, et al. Infant-feeding among low-income women: the social context that shapes their perspectives and experiences. Can J Nurs Res. 2013;45(3):28-49.

9. Sankar MJ, Sinha B, Chowdhury R, et al. Optimal breastfeeding practices and infant and child mortality: a systematic review and meta-analysis. Acta Paediatr. 2015;104(467):3-13.

10. Lodge CJ, Tan DJ, Lau MX, et al. Breastfeeding and asthma and allergies: a systematic review and meta-analysis. Acta Paediatr. 2015;104(467):38-53.

11. Horta BL, Victora CG. Long-term health effects of breastfeeding: a systematic review. OMS; 2013. Disponible en: https://apps.who.int/iris/ handle/10665/79198.

12. Plagemann A, Harder T. Breast feeding and the risk of obesity and related metabolic diseases in the child. Metab Syndr Relat Disord. 2005;3(3):222-32.

13. González de Cosío-Martínez T, Hernández-Cordero S, Rivera-Dommarco J, Hernández-Ávila M. Recomendaciones para una política nacional de promoción de la lactancia materna en México: postura de la Academia Nacional de Medicina. Salud pública Méx. 2017;59(1):106-13

14. Peres KG, Cascaes AM, Nascimento GG, et al. Effect of breastfeeding on malocclusions: a systematic review and meta-analysis. Acta Paediatr. 2015;104(467):54-61.

15. Horta BL, Loret De Mola C, Victora CG. Long-term consequences of breastfeeding on cholesterol, obesity, systolic blood pressure and type 2 diabetes: A systematic review and meta-analysis. Acta Paediatr. 2015;104(467):30-7.
16. Bartick MC, Schwarz EB, Green BD, et al. Suboptimal breastfeeding in the United States: Maternal and pediatric health outcomes and costs. Matern Child Nutr. 2017;13(1):e12366

17. Chowdhury R, Sinha B, Sankar MJ, et al. Breastfeeding and maternal health outcomes: a systematic review and metaanalysis. Acta Paediatr. 2015;104(467):96113.

18. Organisation for Economic Co-operation and Development (OECD). Health at a Glance 2017: OECD Indicators. Paris: OECD; 2017. Disponible en: https://www. oecd-ilibrary.org/social-issues-migrationhealth/health-at-a-glance-2017_health_ glance-2017-en[Consultado el 25 de noviembre del 2020].

19. World Health Organization (WHO). Guideline: protecting, promoting and supporting breastfeeding in facilities providing maternity and newborn services. 2017. Disponible en: https://www.who.int/health-topics/ breastfeeding. [consultado el $25 \mathrm{de}$ noviembre del 2020].

20. World Health Organization (WHO). The optimal duration of exclusive breastfeeding. Report of the expert consultation. Geneva; 2001. Disponible en: https://www.who.int/nutrition/ publications/optimal_duration_of_exc_ bfeeding_report_eng.pdf [Consultado el 15 de noviembre del 2020].

21. World Health Organization (WHO). Global Nutrition Targets 2025: Breastfeeding policy brief. WHO; 2014. Disponible en: https://www.who.int/ nutrition/publications/globaltargets2025 policybrief_breastfeeding/en/ [Consultado el 5 de noviembre del 2020].

22. Ministerio de Desarrollo Social y Familia de Chile. Resultados Encuesta CASEN 2017. Disponible en: http://observatorio. ministeriodesarrollosocial.gob.cl/casenmultidimensional/casen/casen_2017.php [Consultado el 5 de noviembre del 2020].

23. Heymann J, Raub A, Earle A. Breastfeeding policy: A globally comparative analysis. Bull World Health Organ. 2013;91(6):398-406.

24. Gielen AC, Faden RR, O'Campo P, et al. Maternal employment during the early postpartum period: effects on initiation and continuation of breast-feeding. Pediatrics 1991;87(3):298-305.

25. Murtagh L, Moulton AD. Working mothers, breastfeeding, and the law. Am J Public Health. 2011;101(2):217-23.

26. Ministerio de Salud de Chile. Informe técnico. Encuesta Nacional de Lactancia Materna en la Atención Primaria (ENALMA). Chile 2013. Disponible en: https://www.minsal.cl/sites/default/files/
INFORME_FINAL_ENALMA_2013.pdf [Cnsultado el 10 de noviembre del 2020].

27. FAO, FIDA, OPS, WFP y UNICEF. 2020. Panorama de la seguridad alimentaria y nutrición en América Latina y el Caribe 2020. FAO: Santiago de Chile, 2020. Disponible en: https://doi.org/10.4060/ cb2242. [Consultado el 15 de noviembre del 2020].

28. INE. Mujeres en Chile y mercado del trabajo. INE: Santiago, 2015. Disponible en: https://www.ine.cl/docs/defaultsource/ocupacion-y-desocupacion/ publicaciones-y-anuarios/publicaciones/ mujeres-en-chile-y-mercado-deltrabajo---participaci\%C3\%B3nlaboral-femenina-y-brechas-salarialesa. pdf?sfvrsn=ade344d4_3. [Consultado el 15 de noviembre del 2020].

29. Piras Claudia, Rucci Graciana. Chile: Participación laboral femenina y calidad del empleo. Banco Interamericano del Desarrollo (BID): Chile, 2014. Disponible en: https://publications.iadb.org/ publications/spanish/document/ChileParticipaci\%C3\%B3n-laboral-femeninay-calidad-del-empleo.pdf. [Consultado el 15 de noviembre del 2020].

30. Theurich MA, Davanzo R, BusckRasmussen M, et al. Breastfeeding Rates and Programs in Europe: A Survey of 11 National Breastfeeding Committees and Representatives. J Pediatr Gastroenterol Nutr. 2019;68(3):400-7.

31. TRAVAIL legal databases [Internet]. Disponible en: https://www.ilo.org/dyn/ travail/travmain.byCountry2. [citado el 26 de septiembre de 2020].

32. Gatrell CJ. Secrets and lies: Breastfeeding and professional paid work. Soc Sci Med. 2007;65(2):393-404

33. Díaz-Gómez NM, Ruzafa-Martínez M, Ares S, et al. Motivaciones y barreras percibidas por las mujeres españolas en relación a la lactancia materna. Rev Esp Salud Pública 2016;Vol. 90.

34. Boone KM, Dynia JM, Logan J, et al. Socioeconomic Determinants of Breastfeeding Initiation and Continuation for Families Living in Poverty. Pediatrics 2019;144(2 Meeting Abstract):272.

35. Roberts TJ, Carnahan E, Gakidou E. Can breastfeeding promote child health equity? A comprehensive analysis of breastfeeding patterns across the developing world and what we can learn from them. BMC Med. 2013;11(1).

36. Temple Newhook J, Newhook LA, Midodzi WK, et al. Poverty and Breastfeeding: Comparing Determinants of Early Breastfeeding Cessation Incidence in Socioeconomically Marginalized and Privileged Populations in the FiNaL Study. Health Equity. 2017;1(1):96-102. 